\title{
Central nervous system infectious diseases mimicking multiple sclerosis: recognizing distinguishable features using MRI
}

Doenças infecciosas do sistema nervoso central mimetizando esclerose múltipla: reconhecendo as características de imagem por RM que as distinguem Antônio José da Rocha1,2, Ingrid Aguiar Littig1,2, Renato Hoffmann Nunes 1,2, Charles Peter Tilbery³

\begin{abstract}
The current diagnostic criteria for multiple sclerosis (MS) confirm the relevant role of magnetic resonance imaging (MRI), supporting the possibility of characterizing the dissemination in space (DIS) and the dissemination in time (DIT) in a single scan. To maintain the specificity of these criteria, it is necessary to determine whether T2/FLAIR visible lesions and the gadolinium enhancement can be attributed to diseases that mimic MS. Several diseases are included in the MS differential diagnosis list, including diseases with exacerbation, remitting periods and numerous treatable infectious diseases, which can mimic the MRI features of MS. We discuss the most relevant imaging features in several infectious diseases that resemble MS and examine the primary spatial distributions of lesions and the gadolinium enhancement patterns related to MS. Recognizing imaging "red flags" can be useful for the proper diagnostic evaluation of suspected cases of MS, facilitating the correct differential diagnosis by assessing the combined clinical, laboratory and MR imaging information.
\end{abstract}

Keywords: central nervous system infections, multiple sclerosis, differential diagnosis, magnetic resonance imaging, MRI.

RESUMO

Os critérios diagnósticos atuais para a esclerose múltipla (EM) destacam a ressonância magnética (RM) e reforçam a caracterização de disseminação no espaço e no tempo, mesmo em um único exame. Para preservar a especificidade desses critérios é necessário determinar se as lesões identificadas em T2/FLAIR e o realce pelo gadolínio não são devidos a doenças que mimetizam EM. Várias doenças compõem a lista de diagnósticos diferenciais da EM, incluindo algumas com períodos de exacerbação e remissão, além de doenças infecciosas tratáveis, que podem imitar suas características de RM. Discutiremos as características de imagem mais relevantes de diversas neuroinfecções que mimetizam EM, examinando a distribuição espacial das lesões e os padrões de realce pelo gadolínio. O reconhecendo dos sinais de alerta por imagem pode ser útil para a avaliação diagnóstica de casos suspeitos de EM, conduzindo ao diagnóstico diferencial correto através de uma avaliação combinada da clínica, laboratório e imagem.

Palavras-Chave: infecções do sistema nervoso central, esclerose múltipla, diagnóstico diferencial, imagem por ressonância magnética, RM.

The current International Panel on Multiple Sclerosis (MS) Diagnosis (McDonald criteria, 2011) ${ }^{1}$ have confirmed the important role of magnetic resonance imaging (MRI) in the diagnosis of MS, especially for patients who present with clinically isolated syndromes (CIS), because this technique allows the dissemination in space (DIS) and the dissemination in time (DIT) to be characterized in a single scan.
The determination of DIS is based on the demonstration of at least 1 T2/FLAIR lesion in at least two of the four characteristic locations for MS (juxtacortical, periventricular, infratentorial, and spinal cord), excluding the symptomatic lesions in the brainstem or spinal cord (Figure 1). The DIT criteria are based on the histopathological diversity of early MS, in which multifocal chronic plaques often coexist with a few

\footnotetext{
Professor Adjunto do Departamento de Clínica Médica da Faculdade de Ciências Médicas da Santa Casa de São Paulo, São Paulo SP, Brazil; ${ }^{2}$ From the Division of Neuroradiology, Santa Casa de Misericórdia de São Paulo, São Paulo SP, Brazil;

${ }^{3}$ From the Division of Neurology, Santa Casa de Misericórdia de São Paulo, São Paulo SP, Brazil.

Correspondence: Antônio José da Rocha; Santa Casa de Misericórdia de São Paulo / Serviço de Diagnóstico por Imagem; Rua Dr. Cesário Motta Junior 112 / Vila Buarque; 01221-020 São Paulo SP - Brazil; E-mail: a.rocha@uol.com.br

Conflict of interest: There is no conflict of interest to declare. There is no funding source.
} 


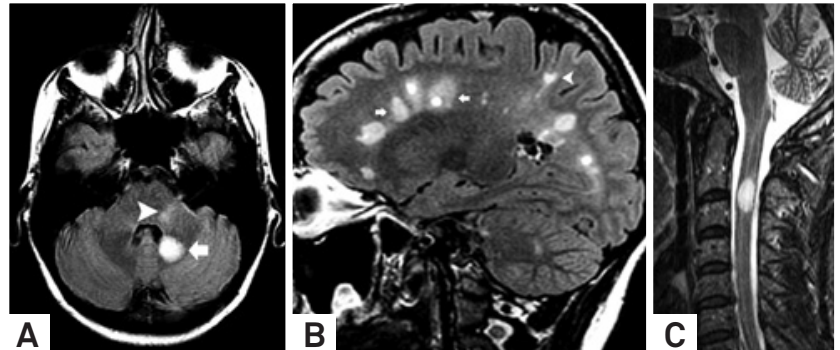

Figure 1. Spatial distribution patterns of lesions included in the current criteria for MS. Axial FLAIR image (A) depicted ovoid hyperintense lesion (arrowhead) in the left intra-axial trigeminal root zone associated with another cerebellar lesion. Sagittal FLAIR image (B) showed characteristics periventricular ("Dawson's fingers") (arrows) and also juxtacortical (arrowhead) lesions. Sagittal T2 WI showed MS lesion in the spinal cord.

areas of inflammation. The current criteria of DIT could be fulfilled if both gadolinium-enhancing $(\mathrm{Gd}+)$ and gadoliniumnonenhancing lesions (Gd-) coexist on the baseline MRL.

One important issue concerning these criteria is related to their specificity. It is necessary to determine whether incipient T2/FLAIR lesions, and particularly whether the $\mathrm{Gd}+$, can be attributable to non-MS diseases ${ }^{1}$. Several CNS diseases are included in the MS differential diagnosis list, primarily those that exhibit exacerbation and remitting periods during its course ${ }^{2}$. Careful interpretation of MRI scans can contribute to an increased awareness of the likelihood of an eventual alternative diagnosis for multifocal lesions and $\mathrm{Gd}+$, highlighting "red flags for imaging MS misdiagnosis"3. Regarding other demyelinating diseases, ischemic lesions, especially microvascular, primary or systemic vasculitis, and genetic or metabolic conditions, the correct diagnosis of MS in developing countries might face additional challenges, including several treatable infectious diseases and sometimes related abnormalities due to prescribed treatments, which can mimic several MRI features of $\mathrm{MS}^{4}$.

This review aims to didactically discuss the most relevant features in several infectious diseases that resemble MS on MRI. This paper examines the primary MS imaging patterns of spatial distribution and Gd enhancement, including optic neuritis (ON) and supratentorial, brainstem, and spinal cord lesions.

\section{SUPRATENTORIAL INFECTIOUS LESIONS THAT MIMIC MS}

\section{Periventricular lesions}

\section{Cytomegalovirus CNS infection}

Cytomegalovirus (CMV) may become latent in cells and have the potential for subsequent reactivation, which is a neurotropism peculiar to the ependyma, germinal matrix, and capillary endothelium. This virus replicates in those specific sites of the inner surface of the ventricles, thereby mimicking MS with typical periventricular distribution. Callosal or periventricular lesions are rare in CNS infections. Ventriculitis occurs in several opportunistic diseases, such as tuberculosis, cryptococosis and, more rarely, toxoplasmosis. CNS primary lymphoma, which is a noninfectious disease, exhibits similar characteristics and is particularly associated with Epstein-Barr virus co-infection. A thin linear abnormal signal on FLAIR and Gd enhancement in the ependimal surface suggest a CMV etiology for ventriculoencephalitis in immunocompromised patients ${ }^{5}$. Ocular infection (retinitis) also corroborates this etiology.

\section{Non-periventricular white matter lesions}

Many infectious diseases will cause white matter brain lesions outside the ventricular surface and corpus callosum, primarily affecting the centrum semiovale and supratentorial regions. Because acute disseminated encephalomyelitis (ADEM) is an autoimmune post-infectious disorder, its description is beyond the scope of the present study.

\section{Toxocariasis}

Toxocariasis human infection (Toxocara canis or Toxocara catis), particularly in children or adolescents, has been reported to be associated with the development of encephalopathy, and its imaging pattern overlaps with that of ADEM, mimicking early onset MS. MRI reveals several subcortical and white matter brain lesions with variable Gd enhancement. The concurrent eosinophilic meningoencephalitis may lead to a hypothesis of toxocariasis ${ }^{6}$.

\section{Neuroborreliosis}

Neuroborreliosis (Borrelia burgdorferi), which is transmitted by ticks, is clinically manifested with erythema migrans that eventually resolves, even without antibiotic treatment. However, the infecting pathogen can spread to other tissues and organs (skin, nervous system, joints, or heart) either hematogenously or retrogradely via the peripheral nerves. Direct CNS symptoms can vary widely and range from a mild confusional state or psychiatric symptoms to severe encephalitis. Neuroborreliosis can cause multifocal and relapsing neurologic symptoms in addition to disseminated white matter lesions in the cerebral MRI ${ }^{7}$ that exhibit several features of MS lesions (Figure 2). The distribution of lesions may occasionally include the callososeptal interface, often without Gd enhancement, which fuels speculation regarding a secondary autoimmune mechanism because it may mimic a primary demyelinating disease ${ }^{8}$.

Despite successful clinical resolution with antibiotic management, white matter involvement often persists on MRI, even in long-term follow-up9. Unlike MS, occult brain and cervical cord pathology in normal-appearing white matter, as 

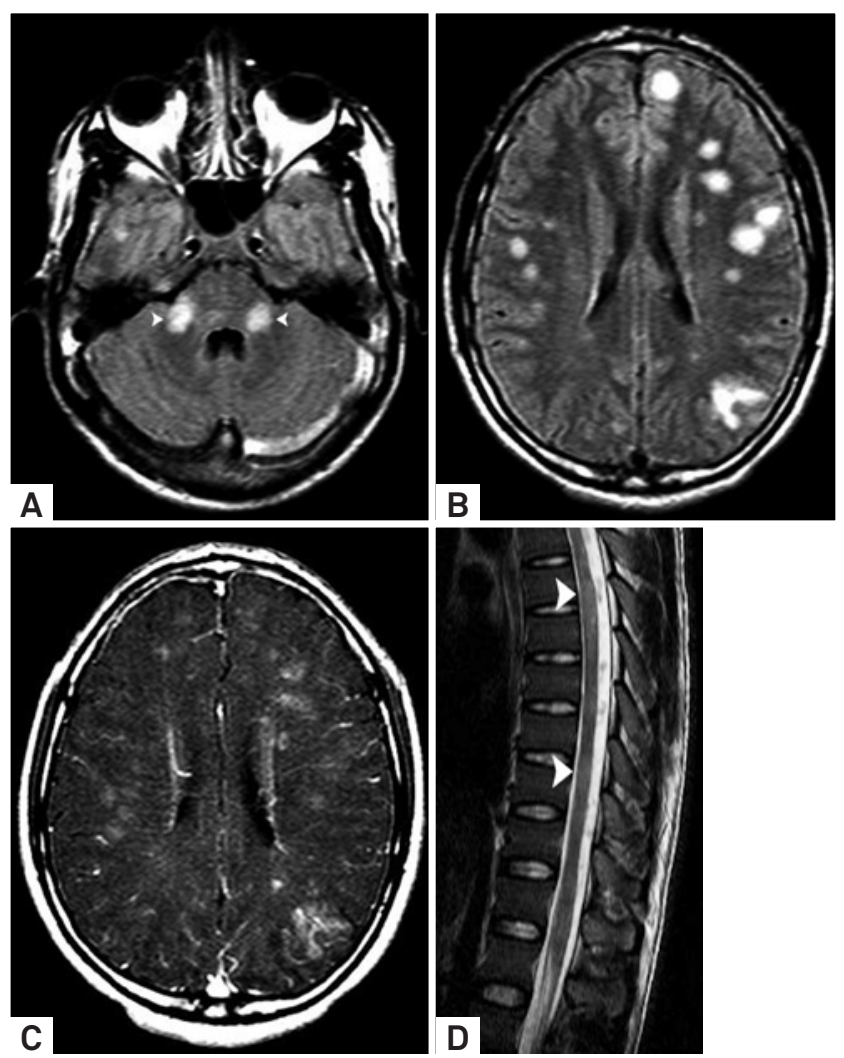

Figure 2. Neuroborreliosis manifesting with disseminated white matter lesions in the brain and spinal cord. Axial FLAIR images (A-B) showed predominant juxtacortical distributed supratentorial lesions and infratentorial involvement of the pontine trigeminal tracts (arrowheads) characterized by pleomorphic (non-ovoid) lesions. Calososeptal interface was not compromised (not shown). Axial T1 WI post-Gd administration (C) showed atypical simultaneous enhancement of most of the lesions (unusual for MS patients). Spinal cord sagittal T2 WI (D) confirmed ovoid hyperintense lesions on thoracic segment (arrowheads).

assessed by magnetization transfer ratios (MTRs) and diffusion tensor imaging, are infrequent findings in patients with neuroborreliosis ${ }^{10}$.

\section{Hepatitis C virus}

Extrahepatic manifestations of HCV infections are well known; the most frequent HCV infections are polyarteritis nodosa, membranoproliferative glomerulonephritis, Sjögren's disease and lymphoma. Nervous system complications are rarely reported and include intracranial vasculopathy, and peripheral neuropathy, which is the neurological disorder most often associated with HCV infection; it is usually combined with type II cryoglobulinemia and described in correlation with multifocal demyelinating mechanisms.

On MRI, the typical imaging characteristics are multiple subcortical and periventricular white matter lesions that are hyperintense on T2/FLAIR images and hypointense on T1, some of them exhibiting an identical pattern of Gd enhancement (Figure 3). The cervical spinal cord and brainstem may
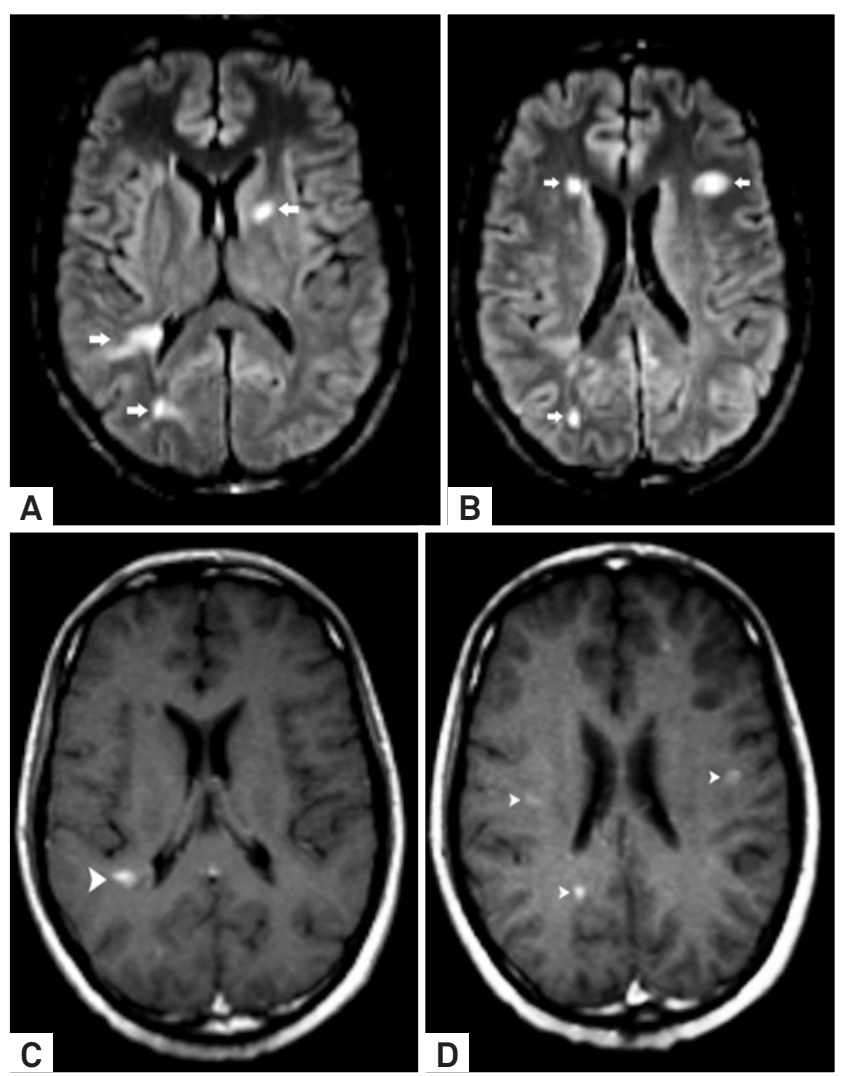

Figure 3. White matter lesions in the hepatitis C virus. Axial FLAIR images (A-B) showed multiple subcortical and periventricular ovoid white matter lesions, mimicking MS features (arrows). Axial T1 WI post-Gd administration (C-D) depicted nodular enhancement in several lesions (arrowheads). Note the absence of the "open-ring" pattern of enhancement.

show similar lesions, increasing the potential of confounding these lesions with MS lesions. Specific serologic reactions, concomitant liver involvement, and peripheral nervous system infection will facilitate distinguishing CNS lesions related to HCV from MS disease ${ }^{11}$.

\section{Other infectious diseases}

Nonenhancing multifocal lesions are usually related to microvascular etiology. However, this pattern of lesions can also be observed in viral CNS diseases, such as multiple punctate hyperintense lesions on T2/FLAIR and diffuse white matter of the cerebral hemispheres, especially in the periventricular and subcortical regions, which exhibit no Gd enhancement and without mass effect. Several virus agents can be associated with viral CNS diseases, such as HTLV-1 and HIV.

"Open ring" enhancement is considered highly specific for demyelinating lesions, particularly in MS, which is useful for differentiating between atypical demyelination and a neoplasm or an abscess. However, several CNS infectious diseases may simulate ring enhancement or "open ring" lesions. 
Neurocysticercosis and occasionally tuberculosis, in some stages or presentations, are associated with MS “open ring”enhanced lesions (Figure 4).

Imaging abnormalities in neurobrucellosis are variable and may also mimic MS brain lesions. The imaging appearance in this disease indicates inflammation, white matter changes with demyelinating processes and vascular insult. Inflammation may cause a granulomatous reaction (Figure 5) or meningeal, perivascular or lumbar nerve root enhancement ${ }^{12}$. Whipple disease is also an uncommon chronic bacterial infection (Tropheryma whipplei) that involves the CNS, sometimes without intestinal manifestation, and leads to multifocal brain abnormalities that may mimic those of MS.

\section{Juxtacortical lesions}

\section{Progressive multifocal leukoencephalopathy (PML)}

PML, which is a demyelinating opportunistic infection of the CNS, is caused by the reactivation of the polyomavirus JC virus (JCV), typically seen in the setting of an
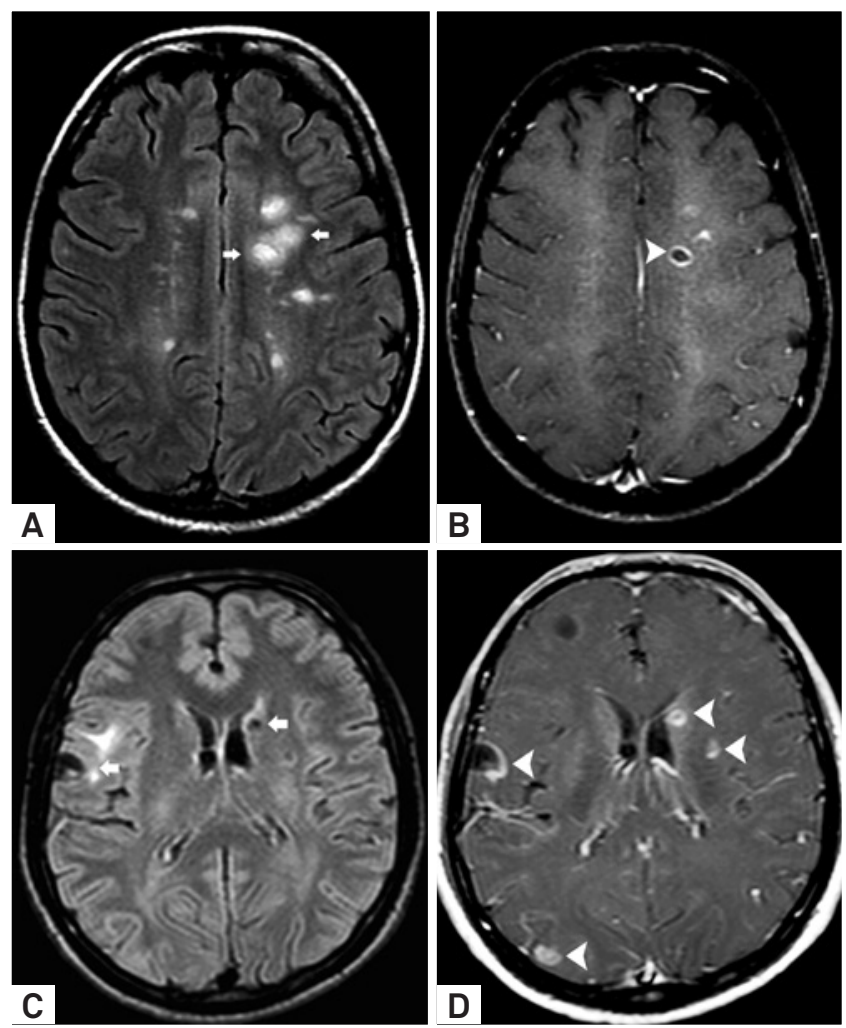

Figure 4. Neurocysticercosis mimicking "open-ring" Gd enhancement. MS typical supratentorial lesions (arrows) hyperintense on FLAIR images (A) and "open-ring" enhancement (arrowhead) was observed on T1 WI post-Gd administration (B). Comparative images in a patient with cysticercosis showed atypical morphology of lesions (arrows) on axial FLAIR (C). Although there was an incomplete annular enhancement in a vesicle on the gray-white matter junction (arrow), there were also several vesicles containing scolex and annular/nodular Gd enhancement (arrowheads) on T1 WI (D). immunocompromised host. The features of PML have expanded considerably since the advent of highly active antiretroviral therapy for HIV patients, leading to the development of immune reconstitution inflammatory syndrome in PML lesions (PML-IRIS).

Recently, monoclonal antibodies (natalizumab) have also increased the incidence of PML. The symptoms of patients with PML-MS are difficult to distinguish from those of patients with MS. As a result, it is crucial to observe imaging aspects that differentiate between new MS lesions and the development of PML concomitant with long-term natalizumab MS treatment ${ }^{13}$. PML lesions are usually diffuse and asymmetric, showing ill-defined borders, which tend to be confluent at late stages of the disease. The lesions are, for the most part, located in the white matter of the brain, typically start in a juxtacortical location and progressively enlarge over weeks, and rarely affect the periventricular white matter in the early stage of the disease (Figure 6). It is assumed that these lesions never affect the optic nerves, rarely appear in the spinal cord, and are often Gd- in immunocompromised patients. Conversely, PML-IRIS lesions typically exhibit multifocal areas that are Gd+. Natalizumab-PML on MRI leads to very destructive cavitated lesions, which are rarely seen in other settings, including patients with $\mathrm{IDS}^{14}$.


Figure 5. Neurobrucellosis. Axial FLAIR images (A-B) showed multiple hyperintense white matter lesions and infra and supratentorial brain lesions (brainstem, corpus callosum and periventricular white matter) suggestive of demyelination (mimicking MS). Note simultaneous Gd enhancement (arrowheads) on T1 WI (C-D). 
Normal-appearing brain tissue (NABT) MTR in PML is usually greater than the NABT MTR in relapsing-remitting MS, which suggests that PML may be more focal than $\mathrm{MS}^{15}$.

\section{Brainstem infectious lesions that mimic MS}

MS lesions in the posterior fossa can present with a multitude of symptoms, including diplopia, trigeminal neuralgia, hypoesthesic facial spasm, deafness, tinnitus, vertigo, dysarthria, dysphagia and ataxia ${ }^{16}$. Infratentorial lesions, a common feature in MS patients, are rarely associated with the presence of black holes that are relevant to differential diagnosis.

The trigeminal nerve, in addition to the intrapontine tract, the pontine root entry zone, the cisternal segment, and the trigeminal nuclei, are characteristic sites of MS lesions. Trigeminal lesions are not uncommon in 3 Tesla MRI (12$38 \%)^{17}$, and thickened trigeminal nerves have been described in MS patients and should be recognized as a rare feature of this disease ${ }^{18}$.

\section{Family herpes virus (HHV)}

Infectious diseases can compromise the brainstem and are often related to family HHV, such as Varicela zoster virus



Figure 6. Imaging follow-up in a renal post-transplantation PML. Axial FLAIR (A-B) images showed infra and supratentorial, asymmetric and confluent hyperintense lesions, predominantly at subcortical regions. Comparative FLAIR images (C-D) after 3 months confirmed lesion confluence and new abnormal foci with similar features, predominantly in the brain hemispheres. There was no evidence of contrast enhancement (not shown).
(VZV) rhombencephalitis, which is a crucial differential diagnosis for trigeminal symptoms with pontine lesions that have a peculiar MRI appearance ${ }^{19}$. In general, all family HHV might affect the pontine root entry zone and the cisternal segment of the trigeminal nerves (Figure 7).

\section{Other infectious diseases}

Dorsal pons and medulla lesions can characterize enterovirus infection (enterovirus 71), which can cause lesions with restricted diffusion of water molecules ${ }^{20}$, and Listeria monocytogenes infection, the most common cause of infectious rhombencephalitis. The latter is transmitted by the consumption of contaminated food ${ }^{21}$ and may mimic the trigeminal nerve involvement of VZV that causes longitudinal posterior lesions through the brainstem.

Other virus infections have been associated with brainstem involvement. Identifying these infections using their MRI features is not easy. However, some features, such as middle cerebellar peduncle involvement or cerebellar atrophy in immunocompromised patients, suggest JCV (rhombencephalic PML or granular neuronopathy). HTLV-1-associated myelopathy causes tropical spastic paraparesis (HAM/TSP) with atrophy in the lower thoracic segment of the spinal cord and is occasionally associated with atypical T2/FLAIR hyperintense brain lesions. Furthermore, the selective involvement of the intracranial corticospinal tracts in HTLV-1 carriers has been described in association with brainstem and middle cerebellar peduncle lesions (Figure 8) ${ }^{22}$.

\section{Optic infectious neuritis that mimics MS}

Optic neuritis (ON) is the most common MS presentation as a CIS. The most recognized clinical red flags that should be considered are as follows: evidence of retinopathy, uveitis, severe continuous orbital pain, persistent complete loss of vision even after a month and macular star on the ophthalmological examination. Additionally, bilateral simultaneous ON, painless and severe disc swelling and retinal hemorrhages are very uncommon features in an MS demyelinating $\mathrm{ON}^{3}$.
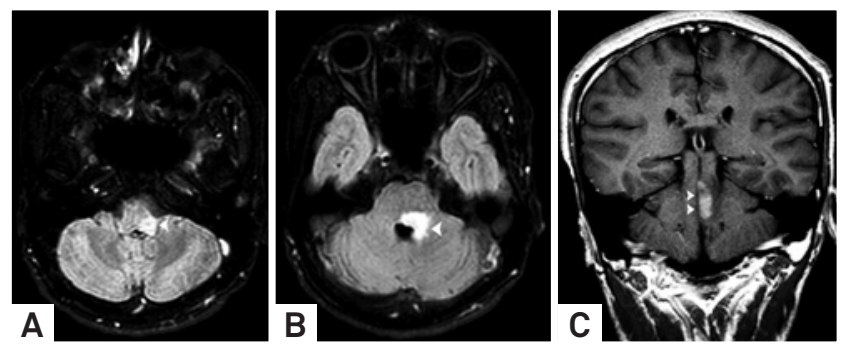

Figure 7. MRI of trigeminal Zoster (VZV). Axial FLAIR images $(A-B)$ revealed abnormal increased signal in the left lower posterior pons and medulla near the fourth ventricle (spinal trigeminal nucleus and tract). Coronal T1 WI postGd intravenous administration (C) demonstrated contrast enhancement along the pontine lesion. 
Although ON is a clinical diagnosis, imaging, especially MRI, plays an important role in confirming the infection and contributing information to determine its prognosis. ON results in edema and it is often associated with long segment of Gd enhancement, which reflects the breakdown of the bloodbrain barrier. Non-arteritic anterior ischemic optic neuropathy and neuromyelitis optica (NMO) are often described as the classical differential diagnoses for MS ON; however, it is important to recognize several infectious diseases that may be important mimickers of demyelinating processes.

\section{Syphilis}

Optic nerve involvement by T. pallidum is often a manifestation of a later stage disease; however, in an immunocompromised patient, neurosyphilis can occur earlier. Optic nerve involvement may be unilateral or bilateral and becomes apparent as perineuritis, anterior or retrobulbar ON or papilloedema. The most common ocular finding in tertiary syphilis is uveitis, which occurs in $2-5 \%$ of patients. In the case of ON, the involvement of other cranial nerves, such as motor ocular

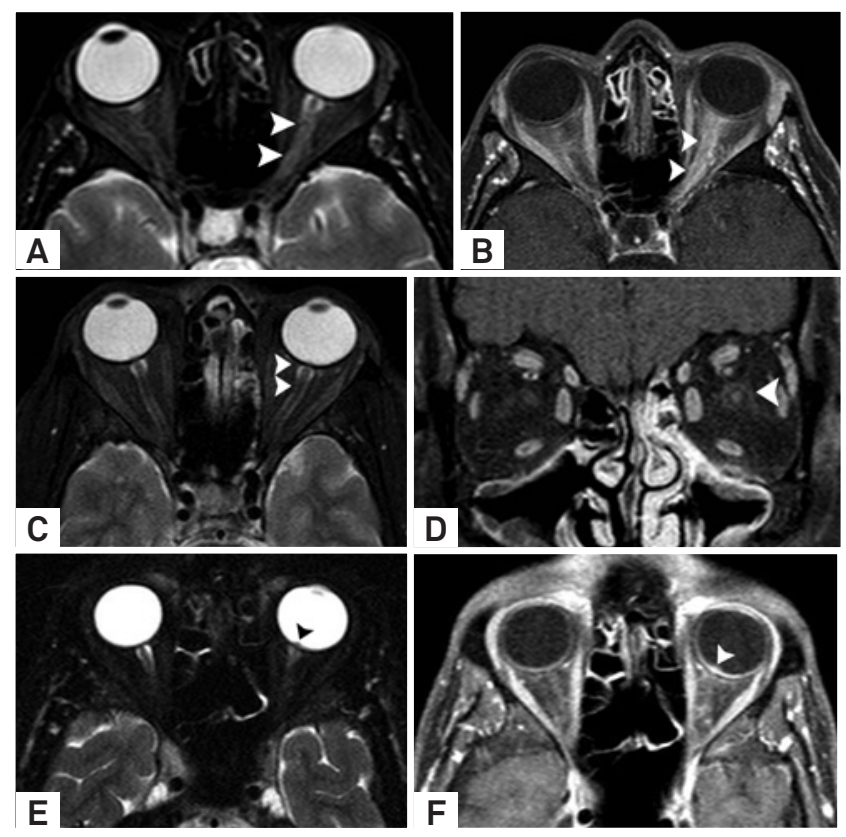

Figure 8. Optic neuritis differential diagnosis. Optic neuritis in MS on axial T2 WI (A) characterized by thickened and hyperintense left optic nerve (arrowheads). Axial T1 WI postGd administration (B) demonstrated contrast enhancement along the orbital segment of the left optic nerve (arrowheads). In another patient with neurosyphilis, comparative images showed papilloedema on left (arrowheads) in T2 WI (C). Coronal T1 WI post-Gd administration revealed a peripheral optic nerve contrast enhancement (D) compatible with left perineuritis and papilitis (arrowhead). In an additional patient with cat scratch disease, comparative images showed a small bulging of the left optic disc (arrowhead) on T2 WI (E). Axial T1 WI postGd administration confirmed short-segment enhancement of the left optic nerve localized to the optic nerve-globe junction (arrowhead). nerves $^{23}$ and vestibulocochlear nerves, which is an extension of the inflammatory processes beyond the optical nerve, are important clues for distinguishing syphilis from ON in MS.

\section{Cat scratch disease}

Cat scratch disease is an infrequent cause of optic neuropathy that is associated with the classic macular star formation on ophthalmological examinations. Orbital MRI shows several characteristic features that should support specific suspicions. Cat scratch disease is characterized by a unilateral short-segment enhancement of the optic nerve localized to the optic nerve-globe junction with less edema in the region of Gd enhancement or in locations other than those that are usually observed in the demyelinating proces$\operatorname{ses}^{24}$. Additionally, bulging of the affected optic disc and extra-axial dural based masses may be observed, increasing the specificity of the imaging findings (Figure 9).

\section{Toxocariasis}

Toxocariasis is an important differential diagnosis, particularly in children with ON suspicion. In ocular larvae migrans, in which pathologic effects on the host are restricted to the eye and the optic nerve, the optic nerve involvement most commonly occurs in its head due to the direct intraocular involvement ${ }^{25}$. In children, the disorder usually occurs as a chronic granulomatous endophthalmitis, which is identified as a vitreous central mass with variable signals on $\mathrm{T} 1$ and $\mathrm{T} 2$ and moderate Gd enhancement, which might be associated with subretinal exudate and retinal detachment. Toxocariasis may be associated with other neurological symptoms, such as eosinophilic meningoencephalomyelitis, encephalitis, extramedullary space-occupying lesion, brain vasculitis, seizures, and most likely behavioral disorder.

\section{Tuberculosis}

Cranial nerve palsies are not rare in CNS tuberculosis $(20-30 \%)$, and the sixth cranial nerve is most commonly


Figure 9. HTLV-I carrier with brainstem signal abnormalities on MRI. Axial comparative images on FLAIR (A-B) showed symmetric involvement of the cerebral peduncle and ventral part of the pons along the corticospinal tracts (arrowheads). Note the predominant asymmetric involvement of the cerebellar peduncles. Note the restricted diffusion of water molecules in these lesions (C). 
involved. Vision loss due to optic nerve damage may occasionally be a dominant symptom. Optochiasmatic arachnoiditis, which is the third ventricular compression of optic chiasma due to hydrocephalus and optic nerve granuloma, is a likely factor for determining clinical manifestations. MRI may reveal choroid tubercles, which are more frequent in tuberculous meningitis associated with miliary tuberculosis and are virtually pathognomonic of tuberculous etiology ${ }^{26}$.

Ethambutol is used to treat tuberculosis; however, it is also associated with permanent visual loss. As ON has been described as one of the toxic effects of ethambutol, it emerges that the drug appears to have a dose-related side effect $^{27}$.

\section{Other diseases}

There are other reports concerning rare causes of ON, such as neuroborreliosis, West Nile virus, Epstein-Barr virus, mycoplasma and herpes virus. The clinical aspects associated with a more extensive ocular impairment and a non-MS radiological picture may suggest a non-MS ON.

\section{Spinal cord lesions that mimic MS}

The spinal cord is involved in more than $90 \%$ of patients with MS. The most well-known clinical red flags include the following: complete transverse myelitis, radiculopathy and areflexia. Additionally, complete Brown-Séquard syndrome, fecal incontinence, cauda equina syndrome, symmetrical progressive spastic paraplegia, acute urinary retention and progressive sensory ataxia should be analyzed ${ }^{3}$.

In contrast to the brain, typical focal, ovoid, spinal cord MS lesions no longer than three vertebral segments in length, usually partially extending into the axial plane, commonly in a dorsolateral location with variable Gd enhancement, with minimal local edema, and without mass effect are rarely found in the spinal cord in other conditions, such as infectious diseases. MS lesions are more common in the cervical segment and are rarely found in the conus medullaris. The identification of T1 hypointense lesions (black holes) is extremely rare. As this pattern of lesions is rare, spinal cord MRI, in addition to brain MRI, may be highly specific for the differential diagnosis of MS.

However, in children, the MS inaugural event may mimic ADEM, which usually shows a more diffuse spinal cord impairment with lesions longer than three vertebral segments in length that frequently occupy more than two-thirds of the cross-sectional area of the cord ${ }^{28}$. In such cases, differentiation from infectious diseases, particularly viral infections, is crucial.

\section{Family herpes virus}

Family herpes virus is a viral agent that is most frequently associated with myelitis. The clinicoradiological presentation is similar, and the etiological diagnosis can be confirmed only by laboratory tests. HHV types 1, 2, 6 and 7 share an overlapping imaging presentation, characterized by long-segment hypersignal on T2 with variable Gd enhancement. There are reports of hemorrhagic lesions, particularly in type $1 \mathrm{HHV}^{29}$. Myelitis is a rare manifestation of VZV infection and generally occurs after reactivation of a latent infection. In VZV myelitis, when a concomitant skin lesion is present (in $33 \%$ of patients), the spinal cord level usually corresponds to the affected dermatome. As in the brainstem, changes are associated with the nerve's apparent origin in the nervous system and are most severe at the dorsal root entry zone and posterior horn of the involved dermatome. Additionally, the MRI may show a variable imaging presentation, including single or multiple lesions, with or without Gd enhancement, usually associated with marked edema ${ }^{30}$. CMV infection is rare in the immunocompetent patient, and imaging usually shows conus medullaris impairment associated with thickening, clumping, and enhancement of nerve roots and leptomeninges, often with associated long-segment cord high signal on T2 (Figure 10).

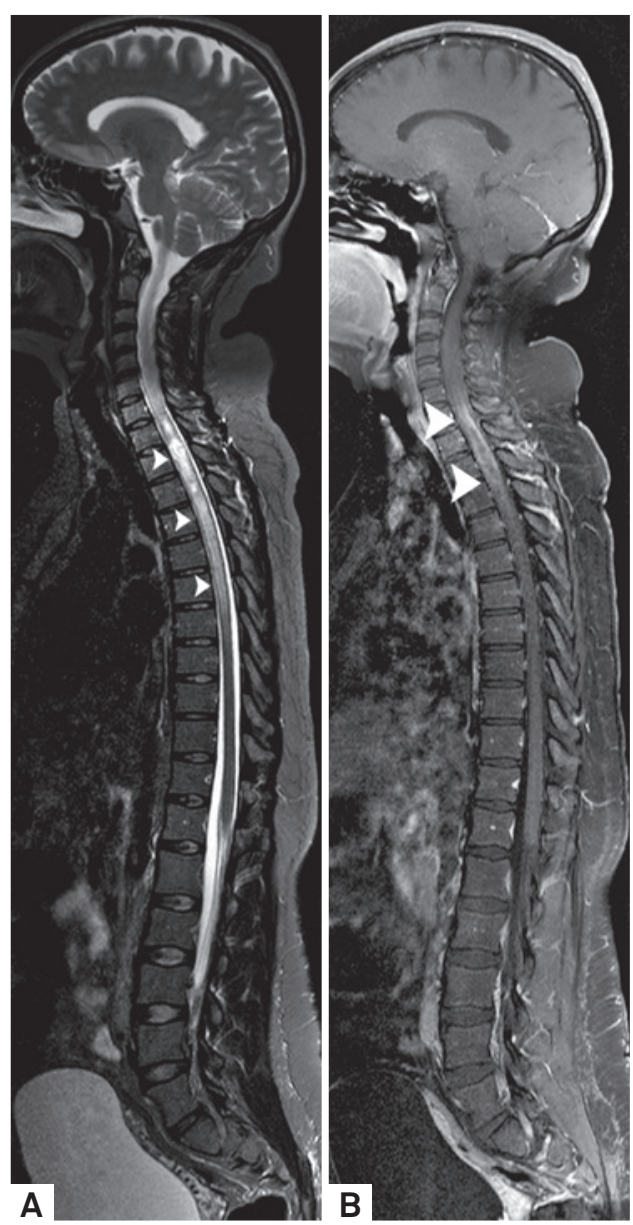

Figure 10. Cytomegalovirus acute myelitis. Sagittal T2 WI (A) and comparative T1 WI post-Gd administration (B) demonstrated central hyperintensity with minimal expansion of long-segment cervico-thoracic lesion in the spinal cord with heterogeneous contrast enhancement. 
Another especially different manifestation of viral diseases is observed in the picornavirus family (poliovirus, enterovirus 71 and, less commonly, Coxsackie virus A and B) and in some flaviviruses, including the West Nile and dengue viruses. This manifestation is marked by a poliomyelitis-related syndrome shown on MRI as a unilateral or bilateral hyperintensity on T2 in the anterior horns of the spinal cord across multiple segments that may be enhanced ${ }^{29}$.

\section{Human T lymphotropic virus type 1 (HTLV-1)}

HTLV-1 is endemic to certain regions and rarely shows neurologic symptoms in its carriers. The spinal cord clinical picture usually reflects impairment of the dorsolateral columns, which can also be identified by MRI as long-segment hyperintensity on T2 of the lateral columns, less commonly extending to the dorsal columns, occasionally with Gd enhancement. It commonly evolves to atrophy of the lateral columns with few signal-intensity abnormalities.

HIV is another potential differential diagnosis for manifestations along the dorsolateral medullary column, which are typically seen over multiple segments in vacuolar myelopathy; however, HIV may also manifest as myelitis with long-segment hyper T2 signal and multifocal Gd enhancement. A similar imaging picture is observed in tabes dorsalis in patients with neurosyphilis, which is usually identified in chronic cases as atrophy of the dorsolateral columns ${ }^{29}$.

\section{Final considerations}

The typical topographical distribution of ovoid T2/ FLAIR hyperintense lesions with "open-ring” Gd enhancement will always favor MS diagnosis in the appropriate setting. Conversely, T2/FLAIR hyperintense non-callosal pleomorphic CNS lesions with an atypical white matter distribution, which are associated with hemorrhage, meningeal involvement, and unusual patterns of Gd enhancement, should suggest "imaging red flags" for possible CNS infections.

Several imaging features are peculiar and facilitate recognizing several infectious diseases and the treatments for CNS complications. However, if nonspecific findings are present, follow-up MRI and appropriate laboratory tests may constitute the best approach.

\section{References}

1. Polman $\mathrm{CH}$, Reingold SC, Banwell B, et al. Diagnostic criteria for multiple sclerosis: 2010 revisions to the McDonald criteria. Ann Neurol 2011;69:292-302.

2. Rolak LA, Fleming JO. The differential diagnosis of multiple sclerosis. The Neurologist 2007;13:57-72.

3. Miller DH, Weinshenker BG, Filippi M, et al. Differential diagnosis of suspected multiple sclerosis: a consensus approach. Mult Scl 2008;14:1157-1174.

4. Brinar VV, Habek M. Rare infections mimicking MS. Clin Neurol Neurosur 2010;112:625-628.

5. Guerini H, Helie O, Leveque C, Adem C, Hauret L, Cordoliani YS. [Diagnosis of periventricular ependymal enhancement in MRI in adults]. J Neuroradiol 2003;30:46-56.

6. Marx C, Lin J, Masruha MR, et al. Toxocariasis of the CNS simulating acute disseminated encephalomyelitis. Neurology 2007;69:806-807.

7. Hahn JS, Pohl D, Rensel M, Rao S, International Pediatric MSSG. Differential diagnosis and evaluation in pediatric multiple sclerosis. Neurology 2007;68(Suppl):S13-S22.

8. Hildenbrand P, Craven DE, Jones R, Nemeskal P. Lyme neuroborreliosis: manifestations of a rapidly emerging zoonosis. Am J Neuroradiol 2009;30:1079-1087.

9. Steinbach JP, Melms A, Skalej M, Dichgans J. Delayed resolution of white matter changes following therapy of B burgdorferi encephalitis. Neurology 2005;64:758-759.

10. Agosta F, Rocca MA, Benedetti B, Capra R, Cordioli C, Filippi M. MR imaging assessment of brain and cervical cord damage in patients with neuroborreliosis. Am J Neuroradiol 2006;27:892-894.

11. Boukhris S, Magy L, Senga-mokono U, Loustaud-ratti V, Vallat JM. Polyneuropathy with demyelinating features in mixed cryoglobulinemia with hepatitis C virus infection. Eur J Neurol 2006; 13:937-941.

12. Al-Sous MW, Bohlega S, Al-Kawi MZ, Alwatban J, McLean DR.
Neurobrucellosis: clinical and neuroimaging correlation. American J Neuroradiol 2004;25:395-401.

13. Bloomgren G, Richman S, Hotermans C, et al. Risk of natalizumabassociated progressive multifocal leukoencephalopathy. N Eng J Med 2012;366:1870-1880.

14. Boster A, Hreha S, Berger JR, et al. Progressive multifocal leukoencephalopathy and relapsing-remitting multiple sclerosis: a comparative study. Arch Neurol 2009;66:593-599.

15. ChenJT, Collins DL, Atkins HL, Freedman MS, Arnold DL. Magnetization transfer ratio evolution with demyelination and remyelination in multiple sclerosis lesions. Ann Neurol 2008;63:254-262.

16. Falini A, Kesavadas C, Pontesilli S, Rovaris M, Scotti G. Differential diagnosis of posterior fossa multiple sclerosis lesions--neuroradiological aspects. Neurol Sci 2001;22 (Suppl 2):S79-S83.

17. Mills RJ, Young CA, Smith ET. Central trigeminal involvement in multiple sclerosis using high-resolution MRI at 3 T. Br J Radiol 2010; 83:493-498.

18. da Silva CJ, da Rocha AJ, Mendes MF, Maia AC, Jr., Braga FT, Tilbery CP. Trigeminal involvement in multiple sclerosis: magnetic resonance imaging findings with clinical correlation in a series of patients. Mult Scl 2005;11:282-285.

19. Aribandi M, Aribandi L. MRI of trigeminal zoster. Neurology 2005; $65: 1812$.

20. Lian ZY, Huang B, He SR, Liang CH, Guo YX. Diffusion-weighted imaging in the diagnosis of enterovirus 71 encephalitis. Acta Radiol 2012;53:208-213

21. Jubelt B, Mihai C, Li TM, Veerapaneni P. Rhombencephalitis / brainstem encephalitis. Curr Neurol Neurosci R 2011;11:543-552.

22. Yata S, Ogawa T, Sugihara S, Matsusue E, Fujii S, Kinoshita T. HTLV-I carrier with unusual brain MR imaging findings. Neuroradiol 2004; $46: 755-758$.

23. O'Connell K, Marnane M, McGuigan C. Bilateral ocular perineuritis 
as the presenting feature of acute syphilis infection. I Neurol 2012;259:191-192.

24. Schmalfuss IM, Dean CW, Sistrom C, Bhatti MT. Optic neuropathy secondary to cat scratch disease: distinguishing MR imaging features from other types of optic neuropathies. Am J Neuroradiol 2005;26:1310-1316.

25. Komiyama A, Hasegawa O, Nakamura S, Ohno S, Kondo K. Optic neuritis in cerebral toxocariasis. J Neurol Neurosurg Psychiatry 1995; 59:197-198.

26. Garg RK. Tuberculosis of the central nervous system. Postgrad Med J 1999;75:133-140.
27. Chen HY, Lai SW, Muo CH, Chen PC, Wang IJ. Ethambutol-induced optic neuropathy: a nationwide population-based study from Taiwan. The Brit J Ophthalm 2012;96:1368-1371.

28. Tenembaum S, Chitnis T, Ness J, Hahn JS. Acute disseminated encephalomyelitis. Neurology 2007;68(Suppl):S23-S36.

29. Goh C, Phal PM, Desmond PM. Neuroimaging in acute transverse myelitis. Neuroim Clin N Am 2011;21:951-973.

30. Gilden DH, Beinlich BR, Rubinstien EM, et al. Varicella-zoster virus myelitis: an expanding spectrum. Neurology 1994;44: 1818-1823. 